\title{
Grid Study on the Basis of Reciprocal Power Losses Calculation
}

\author{
Josifs Survilo* \\ Riga Technical University, Latvia
}

\begin{abstract}
It was found that the losses of some supplierconsumer pairs in a grid (reciprocal losses) can be negative. Upon exploring this issue, it turned out that this phenomenon is not rare and is a result of a certain distribution of the power of electricity suppliers between consumers. Negative reciprocal losses indicate that the overall grid losses are increased. They can be eliminated by redistributing the suppliers' power. Computing the reciprocal losses, additional properties of a power system can be defined, which facilitates the determination of its more economical operation mode.
\end{abstract}

Keywords - Node voltage, power grid, power loss, reciprocal current, vector-matrix.

\section{INTRODUCTION}

The price of electricity depends on many factors, to recall the main of these: the suppliers' or power plants' price, the cost of management, administration and maintenance of the infrastructure, support of renewable energy [1] as well as power losses. The choice of suppliers primarily depends on the price and amount of electricity bought [2] whereas power losses have a secondary role.

Once the suppliers have been selected, optimization of the power system mode is a permanent effort of those dealing with electricity. Now, attention is paid to power losses: computer programs are dedicated to optimal power flow that reduces losses [3], various scientific literature has long been devoted to this subject, researching issues related to losses of electricity transfer, grid development, construction and operation [4], [5]. Reference [6] directly links the economy with losses.

Of particular interest is allocation of power losses, in other words, reciprocal losses (RL), which makes it possible to apply more equitable compensation for electricity and to set fairer overheads.

This issue is receiving a lot of attention. Here are some examples of the proposed methods of load allocation and their shortcomings.

In [7], the supplier-consumer losses are not found directly but in a roundabout manner, i.e. by summation. Power allocation exclusively for radial grids is considered in [8]. A comparison of different loss allocation algorithms is made in [9]. In the literature, one can see cumbersome formulas that depend on grid configuration.

The method of loss allocation proposed in [10]-[13] is more accurate and is uniform for all grid configurations. It relies on the reciprocal current (RC), which is needed to transport a portion of some supplier power (reciprocal power - RP) to certain a consumer. Accompanying RL are defined; besides, sometimes these losses are negative.

The goal of the paper is to study how often the negative RL appear and what is the reason of their occurrence; moreover, how the proposed method of load allocation can be used for deeper characterization of the grid.

Section 2 provides clarification of the formula for partial loss determination. In Section 3, a concise algorithm for computing $\mathrm{RL}$ is described and the cause of negative RL is clarified. In Section 4, partial losses and voltage drops in grid elements are considered. In Section 5, the feature to minimize the power losses is considered. Quick determination of voltages at grid nodes is elaborated in Section 6. Conclusions are stated in Section 7.

Voltage, current and power are given in volts, amperes and watts, impedance - in ohms and they are complex quantities unless otherwise shown.

\section{FoRMUlA FOR PARTIAL POWER LOSSES}

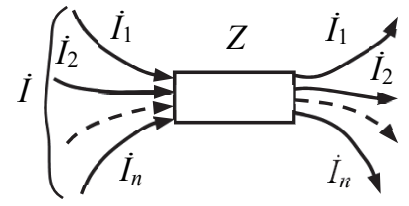

Fig. 1. Impedance $Z$ loaded with a number of currents.
If several currents flow through some impedance (Fig. 1), then the power loss in this impedance caused by some of these currents, e. g. current $I_{2}$, is here called partial loss. Based on publications [10]-[13] and Fig. 5, such loss is defined by the following formula:

$\Delta s_{2}=k \frac{Z}{4}\left(\left|\dot{I}+\frac{\dot{I}_{2}}{k}\right|^{2}-\left|\dot{I}-\frac{\dot{I}_{2}}{k}\right|\right)=\frac{1}{\kappa} \frac{Z}{4}\left(\left|\dot{I}+\kappa \dot{I}_{2}\right|^{2}-\left|\dot{I}-\kappa \dot{I}_{2}\right|^{2}\right)$.

For convenience in further consideration, substitution $k=1 / \kappa$ is made. In [13] $k=100$, then

$$
\Delta s_{2}=25 Z\left(\left|\dot{I}+0.01 \dot{I}_{2}\right|^{2}-\left|\dot{I}-0.01 \dot{I}_{2}\right|^{2}\right) \text {. }
$$

To substantiate a simpler formula, complex numbers are presented as follows:

$$
\dot{I}=I(\cos \alpha+j \sin \alpha) ; \quad \dot{I}_{2}=I_{2}(\cos \beta+j \sin \beta) .
$$

Inserting (2) into expression $c=\left|\dot{I}+\kappa \dot{I}_{2}\right|^{2}-\left|\dot{I}-\kappa \dot{I}_{2}\right|^{2}$, we obtain the following:

\footnotetext{
* Corresponding author.

E-mail address: survilo@eef.rtu.lv
} 


$$
c=4 \kappa I I_{2}[\cos (\alpha-\beta)]=4 \kappa I I_{2}(\cos \alpha \cos \beta+\sin \alpha \sin \beta) .(3)
$$

Inserting (3) into (1), we obtain:

$$
\Delta s_{2}=Z I I_{2}(\cos \alpha \cos \beta+\sin \alpha \sin \beta) .
$$

Whatever number we have assigned to $k$ or $\kappa$ in (1), we still get (4). When we insert $k=1$ into (1), we have

$$
\Delta s_{2}=0.25 Z\left(\left|\dot{I}+\dot{I}_{2}\right|^{2}-\left|\dot{I}-\dot{I}_{2}\right|^{2}\right) .
$$

Reduction $k$ times of partial current (it was reciprocal current) was necessary at the very beginning [10], searching for RP.

The sum of partial power losses, which are computed by formulas based on expression (1), is equal to the power loss caused by the summary current (which corresponds to the essence of electrical engineering):

$$
\Delta S=Z I^{2}=\Delta s_{1}+\Delta s_{2}+\ldots+\Delta s_{n} .
$$

The formula of other type of partial losses is the sum of losses equaled to summary current loss.

\section{RECIPROCAL LOSSES}

The research is being conducted on a $330 \mathrm{kV}$ radial grid (Fig. 2) and on a closed grid (Fig. 3).

For simplification purposes, permittance is assumed zero. In [12], it is shown how power line admittances can be dealt with.

Twenty cases with adequate node power in accordance with Table I are considered to facilitate and illustrate the task.

For each case, the RL $\Delta s_{j t k}$ has to be computed first, where $\Delta s_{j t k}$ is the power spent for transmitting a portion of electricity (the above-mentioned RP) from a specified supplier ' $j$ ', which reaches (in the index, the letter ' $t$ ' stands for 'to') a certain consumer ' $k$ '. The principal quantity for computing RL and other quantities including RP, is reciprocal current RC [13], the current which delivers RP from supplier ' $j$ ' to consumer ' $k$ '.

In this paper, the algorithm of for the determination of RL is called the double algorithm (DA) since it involves the use of two computer programs: any power flow program (here, Power World) and Matlab. The DA is described just further.

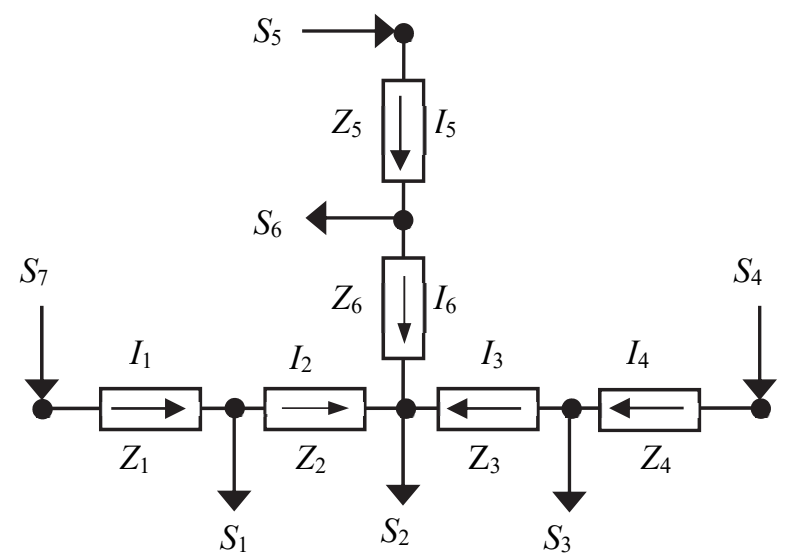

Fig. 2. Circuit diagram of the radial grid (Q). $S_{4}, S_{5}, S_{7}$ - flows to powersupplying points; $S_{1}, S_{2}, S_{3}, S_{6}$ - flows to consumers; $I$ - branch currents; $Z$ transmission line impedances: $Z_{1}=(0.785+3.2 i) \Omega, Z_{2}=(5.58+28.8 i) \Omega$, $Z_{3}=(7.425+24 i) \quad \Omega, \quad Z_{4}=(1.782+5.76 i) \quad \Omega, \quad Z_{5}=(3.465+11.2 i) \quad \Omega$,

\begin{tabular}{|c|c|c|c|c|c|c|}
\hline \multirow{2}{*}{ Case } & $S_{1}$ & $S_{2}$ & $S_{3}$ & $S_{4}$ & $S_{5}$ & $S_{6}$ \\
\hline & \multicolumn{6}{|c|}{ MW, Mvar $i$} \\
\hline Qa & & & & $100+14 i$ & $50+5 i$ & \\
\hline Qc & & & & $75+8.5 i$ & $75+8.5 i$ & \\
\hline Qc1 & & & & $60+6.8 i$ & $90+10.2 i$ & \\
\hline Qc2 & & & & $67.5+7.65 i$ & $82.5+9.35 i$ & \\
\hline Q2i & & $208+39 i$ & & & & \\
\hline Q2i3 & & $256+48 i$ & & & & \\
\hline Q2r & & $100+100 i$ & & & & \\
\hline Q4i3 & & & & $160+50 i$ & & \\
\hline Q4r & & & & $70+50 i$ & & \\
\hline $\mathrm{Fa}$ & & & & $100+14 i$ & 40 & \\
\hline $\mathrm{Fc}$ & & & & $56+5.6 i$ & $84+8.4 i$ & \\
\hline $\mathrm{Fc} 1$ & & & & $42+4.2 i$ & $58+5.8 i$ & \\
\hline $\mathrm{Fc} 2$ & & & & $25.8+2.58 i$ & $34.2+3.42 i$ & \\
\hline $\mathrm{Fc} 3$ & & & & $18.48+1,848 i$ & $23.52+2.35 i$ & \\
\hline $\mathrm{Fc} 4$ & & & & $12.43+1.243 i$ & $15.57+1.57 i$ & \\
\hline Fc5 & & & & $15.68+1.568 i$ & $19.32+1.93 i$ & \\
\hline $\mathrm{F} 2 \mathrm{~d}$ & & $50+20 i$ & & & & \\
\hline $\mathrm{F} 2 \mathrm{r}$ & & $40+100 i$ & & & & \\
\hline F4w6 & & & & 0 & & \\
\hline F4w4 & & & & $50+7 i$ & & \\
\hline
\end{tabular}
$Z_{6}=(3.366+10.88 i) \Omega$.
TABLE I

LOAD OF POWER GRID NODES

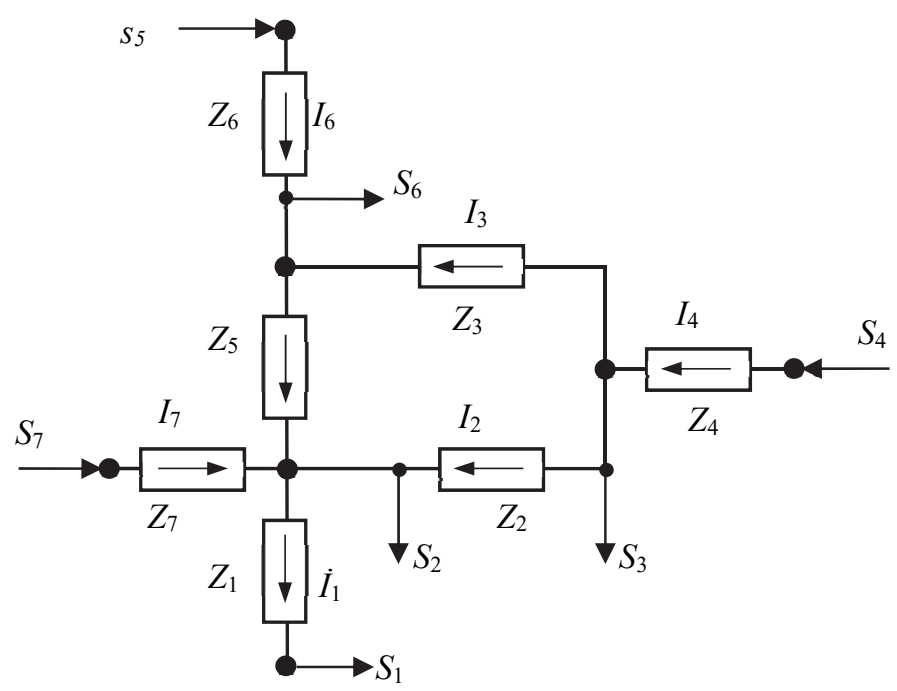

Fig. 3. Circuit diagram of the closed grid (F)

$S_{4}, S_{5}, S_{7}$ - flows to power supplying points; $S_{1}, S_{2}, S_{3}, S_{6}$ - flows to consumers; $I$ - branch currents; $Z$ - transmission line impedances:

$\left.Z_{1}=(11.22+70.4 i) \Omega, Z_{2}=6.1+32 i\right) \Omega, Z_{3}=(6.48+25.6 i) \Omega, Z_{4}=11.125+$ 80i) $\Omega, Z_{5}=(1.02+6.4 i) \Omega, Z_{6}=(10.2+64 i) \Omega, Z_{7}=(0.81+3.2 i) \Omega$.

Briefly, using the circuit diagram shown in Fig. 2, to determine RL, the following should be done: 1) using Power World, determine node voltages $U_{1} \ldots U_{7}$ and their angles $\alpha_{1} \ldots \alpha_{7}$ relative to the slack bus 7 (the indices of the voltages and nodes coincide with those of node power $S$ ); 2) determine node currents $J_{1} \ldots J_{7}$ by means of Matlab; 3) determine branch admittances for the studied grid, $Y_{1} \ldots Y_{6} ; 4$ ) determine consumer 
admittances $Y_{\mathrm{c} 1}, Y_{c 2}, Y_{c 3}, Y_{c 6}$ by means of Matlab; 5) build conductance matrix $\boldsymbol{Y}$ and its inverse matrix $\boldsymbol{Z}_{\mathrm{v}}$;6) build matrix $\boldsymbol{A}$ and its inverse matrix $\boldsymbol{B}$; 7) build matrix $\boldsymbol{Z} ; 8$ ) build node current vector-matrix $\boldsymbol{J}_{\mathrm{v} 4}, \boldsymbol{J}_{\mathrm{v} 5}, \boldsymbol{J}_{\mathrm{v} 7}$ to determine the node voltages; 9) determine supplier-consumer phase node voltages $U_{\mathrm{v} 4 \mathrm{t} 1 \ldots} U_{\mathrm{v} 7 \mathrm{t} 6}$ to compute $\left.\mathrm{RC} ; 10\right)$ perform calculations according to formula batch (7):

$I_{4 \mathrm{t} 1}=Y_{c 1} U_{4 \mathrm{t} 1}, \ldots, I_{7 \mathrm{t} 6}=Y_{c 6} U_{7 \mathrm{t} 6} ; J_{4 \mathrm{i} 4 \mathrm{t} 1}=J_{4}+I_{4 \mathrm{t} 1} ; J_{1 \mathrm{i} 4 \mathrm{t} 1}=J_{1}+I_{4 \mathrm{t} 1}$; $\boldsymbol{J}_{\mathrm{i} 4 \mathrm{t} 1}=\left[\begin{array}{llllll}-J_{1 \mathrm{i} 4 \mathrm{t} 1}, & -J_{2}, & -J_{3}, & J_{4 \mathrm{i} 4 \mathrm{t} 1}, & J_{5}, & -J_{6}\end{array}\right] ; \quad \boldsymbol{I}_{\mathrm{i} 4 \mathrm{t1}}=\boldsymbol{B} \boldsymbol{J}_{\mathrm{i} 4 \mathrm{t} 1} ;$ $\Delta s_{\mathrm{i} 4 \mathrm{t} 1}=\boldsymbol{I}_{\mathrm{i} 4 \mathrm{t} 1}{ }^{\prime} \boldsymbol{Z} \boldsymbol{I}_{\mathrm{i} 4 \mathrm{t} 1} ; \quad J_{4 \mathrm{~d} 4 \mathrm{t} 1}=J_{4}-I_{4 \mathrm{t} 1} ; \quad J_{1 \mathrm{~d} 4 \mathrm{t} 1}=J_{1}-I_{4 \mathrm{t} 1} ; \quad \boldsymbol{J}_{\mathrm{d} 4 \mathrm{t} 1}=\ldots ;$ $\boldsymbol{I}_{\mathrm{d} 4 \mathrm{t} 1}=\ldots ; \Delta s_{\mathrm{d} 4 \mathrm{t} 1}=\ldots ; \Delta s_{4 \mathrm{t} 1}=0.25\left(\Delta s_{\mathrm{i} 4 \mathrm{t} 1}-\Delta s_{\mathrm{d} 4 \mathrm{t} 1}\right) ; \ldots ; \Delta s_{7 \mathrm{t} 6}=\ldots ;$ $s \Delta s=\Delta s_{4 \mathrm{t} 1}+\ldots+\Delta s_{7 \mathrm{t} 6} ; \quad \boldsymbol{J}=\left[\begin{array}{lllll}-J_{1}, & -J_{2}, & -J_{3}, J_{4}, & J_{5}, & -J_{6}\end{array}\right] ;$

$$
\boldsymbol{I}=\boldsymbol{B} \boldsymbol{J} ; \Delta \mathrm{s}=\boldsymbol{I} \boldsymbol{Z I} ; \boldsymbol{J}_{\mathrm{v}}=\left[0,0,0, J_{4}, J_{5}, 0, J_{7}\right] ; \boldsymbol{U}_{\mathrm{v}}=\boldsymbol{Z}_{\mathrm{v}} \boldsymbol{J}_{\mathrm{v}},
$$

where $I_{4 \mathrm{t} 1} ; \ldots ; I_{7 \mathrm{t} 6}$ are the RCs for all the pairs between suppliers and consumers; $J_{4 \mathrm{i} 4 \mathrm{t} 1}$ - node current of supplier 4 incremented by RC $I_{4 \mathrm{t} 1} ; J_{1 \mathrm{i} 4 \mathrm{t} 1}$ - the same for consumer node $1 ; \boldsymbol{J}_{\mathrm{i} 4 \mathrm{t} 1}$ incremented node 4 current vector; $\boldsymbol{I}_{\mathrm{i} 4 \mathrm{t} 1}$-branch current vector (the currents in all branches) of node 4 incremented by $I_{4 \mathrm{t} 1}$; $\boldsymbol{I}_{\mathrm{i} 4 \mathrm{t} 1}{ }^{\prime}$ - conjugate transposed current vector $\boldsymbol{I}_{\mathrm{i} 4 \mathrm{t} 1} ; \Delta s_{\mathrm{i} 4 \mathrm{t} 1}$-power loss for one phase in the grid, incremented by RC $I_{4 \mathrm{t} 1} ; J_{4 \mathrm{~d} 4 \mathrm{t} 1}-$ node current of supplier 4 decremented by RC $I_{4 \mathrm{t} 1} ; J_{1 \mathrm{~d} 4 \mathrm{t} 1}-$ the same for consumer node $1 ; \ldots ; \Delta s_{4 \mathrm{t} 1}-\mathrm{RL}$ for one phase of supplier 4 to consumer $1 ; s \Delta s-$ sum of RC for one phase of all supplier-consumer pairs; I - branch current vector; $\Delta s$ - total losses of the grid; $\boldsymbol{J}$ - supplier node current vector; $\boldsymbol{U}_{\mathrm{v}}-$ node phase voltage vector.

The size of the paper does not allow a more exhaustive description of the algorithm. Details are to be found in [10][13]. However, the best idea about DA can be got by acquainting oneself with the Matlab file.

To make sure that no errors have not found their way into the DA calculations, the sum of the RLs, $s \Delta s$, should be compared with total grid loss $\Delta s$; at least four to five digits should match.

Specific data calculated for case Qa are given in Table II and losses - in Table III.

Total losses $\Delta s$ and sum of RL, $s \Delta s$, are as follows in Qa: $\Delta s=337130+1259600 i ; s \Delta s=337140+1259600 i$.

\section{TABLE II}

Node CURRENTS J, Voltages U U AND BRANCH CURRENTS I For CASE Qa

\begin{tabular}{|c|c|c|c|}
\hline No & $J_{1 \ldots 7}$ & $U_{\mathrm{v} 1 \ldots 7}$ & $I_{1 \ldots 6}$ \\
\hline 1 & $70.09-0.2 i$ & $190234.4-521.7 i$ & $176.73-48.58 i$ \\
\hline 2 & $282.29-58.1 i$ & $188245.9-3322.9 i$ & $106.64-48.38 i$ \\
\hline 3 & $35.19-17.6 i$ & $189435+3.1 i$ & $140.47-6.09 i$ \\
\hline 4 & $175.66-23.69 i$ & $189884.5+972.7 i$ & $175.66-23.69 i$ \\
\hline 5 & $88.16-9.77 i$ & $188818.6-1998.7 i$ & $88.16-9.77 i$ \\
\hline 6 & $52.98-6.14 i$ & $188403.7-2952.3 i$ & $35.18-3.63 i$ \\
\hline 7 & $176.73-48.58 i$ & $190528.6+5.7 i$ & \\
\hline
\end{tabular}

TABLE III

RL ON THE PATH FROM SUPPLIER 4, 5, 7 TO CONSUMER 1, 2, 3, 6 OF CASE Qa

\begin{tabular}{|c|c|c|c|}
\hline $\begin{array}{c}\text { Con- } \\
\text { sumer }\end{array}$ & $\begin{array}{c}\text { Supplier } 4 \\
\Delta s_{411,2,3,6}\end{array}$ & $\begin{array}{c}\text { Supplier } 5 \\
\Delta s_{51,2,3,6}\end{array}$ & $\begin{array}{c}\text { Supplier } 7 \\
\Delta s_{7 \mathrm{t} 1,2,3,6}\end{array}$ \\
\hline 1 & $20934+36622 i$ & $-2195-22479 i$ & $4086+16658 i$ \\
\hline 2 & $154210+498440 i$ & $24360+78740 i$ & $93347+463380 i$ \\
\hline 3 & $4851+15681 i$ & $-4387-14181 i$ & $-1868+15691 i$ \\
\hline 6 & $26081+84302 i$ & $3270+10570 i$ & $14449+76158 i$ \\
\hline
\end{tabular}

For case $\mathrm{Fa}$, the above-mentioned types of data are given in Table IV and $\mathrm{V}$.

In Fa: $\Delta s=552510+3581100 i ; s \Delta s=552510+3581100 i$.

So, the radial grid Qa has even three types of negative reciprocal losses. To find out the reason of negative RL, let us calculate RL $\Delta S_{5 t 3}$ of case Qa, transmitting the electricity from supplier 5 to consumer 3 . This is done without applying matrix algebra, using the current values calculated by DA. RC flows through the impedances $Z_{3}, Z_{5}, Z_{6}$. These currents in the abovementioned impedances are as follows: $I_{31573}=-7.0907+3.0637 i$; $I_{51573}=7.0907-3.0637 i ; I_{6 I 573}=7.0907-3.0637 i$. Through $Z_{1}, Z_{2}$, $Z_{4}$, RC $I_{5 \mathrm{t} 3}$ does not flow. The total current in $Z_{3}, Z_{5}, Z_{6}$ is as follows: $I_{3}=140.47-6.09 i ; I_{5}=88.16-9.77 i ; I_{6}=35.18-3.63 i$. RL $\Delta S_{5 \mathrm{t} 3}$ is the sum of the partial losses in impedances $Z_{3}, Z_{5}$, $Z_{6}$. Observing (5), we have the following: $\left.\Delta S_{3 \mathrm{i} 5 \mathrm{t} 3}=\left.0.25 Z_{3}\left(\mid I_{3}+I_{3 \mathrm{i} 5 \mathrm{t} 3}\right)\right|^{2}-\left|I_{3}-I_{3 \mathrm{i} 5 \mathrm{t} 3}\right|^{2}\right) ; \ldots ; \ldots$. Their values are: $\quad \Delta s_{3 \mathrm{i} 5 \mathrm{t} 3}=-7534-24353 i ; \quad \Delta s_{5 \mathrm{i} 5 \mathrm{t} 3}=2269+7335 i$; $\Delta s_{6 \mathrm{i} 5 \mathrm{t} 3}=877+2835 i$, the sum of which is $\Delta s_{5 \mathrm{t} 3}=-4388-14183 i$. The RL in impedance $Z_{3}$ is negative because current $I_{3 \mathrm{i} 5 \mathrm{t} 3}$ flows in the direction opposite to total current $I_{3}$.

TABLE IV

NODE CURRENTS J, VOLTAGES Uv AND BRANCH CURRENTS I FOR CASE FA

\begin{tabular}{|c|c|c|c|}
\hline No & $J_{1 \ldots 7}$ & $U_{\mathrm{v} 1 \ldots 7}$ & $I_{1 \ldots 6}$ \\
\hline 1 & $70.38-2.03 i$ & $189277.3-5461.4 i$ & $70.38-2.03 i$ \\
\hline 2 & $280,24-53.36 i$ & $190210-529.1 i$ & $79.69-5.13 i$ \\
\hline 3 & $17.48-1.56 i$ & $190860.1+1989.7 i$ & $75.83-3.38 i$ \\
\hline 4 & $173-10.07 i$ & $193589.9+15717.8 i$ & $173-10.06 i$ \\
\hline 5 & $52.56-5.24 i$ & $190282.3+70.3 i$ & $93.09+3.53 i$ \\
\hline 6 & $69.81+1.67 i$ & $190887.5+4555.1 i$ & $69.81+1.67 i$ \\
\hline 7 & $177.85-53.79 i$ & $190526.2-3.5 i$ & $177.85-53.79 \mathrm{i}$ \\
\hline
\end{tabular}

TABLE V

RL ON THE PATH FROM SUPPLIER 4,5,7 TO CONSUMER 1, 2, 3, 6 FOR CASE FA

\begin{tabular}{|c|c|c|c|}
\hline $\begin{array}{c}\text { con- } \\
\text { sumer }\end{array}$ & $\begin{array}{c}\text { supplier } 4 \\
\Delta s_{4 \mathrm{t} 1,2,3,6}\end{array}$ & $\begin{array}{c}\text { supplier } 5 \\
\Delta s_{5 \mathrm{t} 1,2,3,6}\end{array}$ & $\begin{array}{c}\text { supplier } 7 \\
\Delta s_{7 \mathrm{t} 1,2,3,6}\end{array}$ \\
\hline 1 & $92800+609230 i$ & $18155+114630 i$ & $28654+169110 i$ \\
\hline 2 & $286120+1905100 i$ & $37304+237040 i$ & $18750+74074 i$ \\
\hline 3 & $13868+99726 i$ & $758+7274 i$ & $-868-14510 i$ \\
\hline 6 & $51199+340240 i$ & $6186+38814 i$ & $1585+442 i$ \\
\hline
\end{tabular}


The precondition for negative RL lies in opposite directions of the total current in some impedance and its reciprocal current. When the sum of losses is negative, we have negative RL. Negative power loss in any impedance causes an increase of the total losses in a grid.

\section{PARTIAl QuANTITIES IN THE ElEMENTS OF A GRID}

Partial power losses caused by a node current or a reciprocal current can be computed in any line or transformer. This can also be done for voltage drop. It is shown using case Qa.

The currents $I_{1 \ldots 6}$ in lines $1 . . .6$ (the line or branch numbers coincide with the impedance indices in Fig. 2) caused by the currents of a certain node, be it a supplier or a consumer (here, the currents caused by node 4):

$$
I_{1 \ldots 6, J 4 \mathrm{o}}=\boldsymbol{B J}_{4 \mathrm{o}} ; \boldsymbol{J}_{4 \mathrm{o}}=\left[-I_{4 \mathrm{t} 1},-I_{4 \mathrm{t} 2},-I_{4 \mathrm{t} 3}, J_{4}, 0,-I_{4 \mathrm{t} 6}\right] .
$$

These currents are as follows: $I_{1 J 40}=0$; $I_{2 J 40}=-0.2735-0.0067 i ; \quad I_{3 J 40}=1.6096-0.1781 i$; $I_{4 J 40}=1.7566-0.2369 i ; I_{5 J 40}=0 ; I_{6 J 40}=-0.2099+0.0142 i$.

The power loss of one phase in a branch, for example, branch 2, caused by any node (in this case, node 4), is as follows:

$$
\Delta s_{2 J 40}=0.25 Z_{2}\left(\left|I_{2}+I_{2 J 40}\right|^{2}-\left|I_{2}-I_{2 J 40}\right|^{2}\right),
$$

where $I_{2}$ is the current in branch 2 computed by formula $\boldsymbol{I}=\boldsymbol{B} \boldsymbol{J}$ in batch (7).

The calculated losses in branches $2, \ldots, 6$, caused by supplier 4, are as follows: $\Delta s_{2 J 40}=-16094-83064 i$; $\Delta s_{3 J 4 \mathrm{o}}=168680+543240 i ; \quad \Delta s_{4 J 4 \mathrm{o}}=55986+180970 i ;$ $\Delta s_{6 J 40}=-2502.9-8090.2 i$.

The losses in a particular line (any of lines 1...6) as a result of some RCs are calculated in a similar way; let us consider the currents in lines 1 ...6 caused by RC $I_{4 \mathrm{t} 1}$ :

$$
I_{1 \ldots 6 \mathrm{i} 4 \mathrm{t} 1}=\boldsymbol{B} \boldsymbol{J}_{\mathrm{i} 4 \mathrm{t} 1} ; \boldsymbol{J}_{\mathrm{i} 4 \mathrm{t} 1}=\left[-I_{4 \mathrm{t} 1}, 0,0, I_{4 \mathrm{t} 1}, 0,0\right] .
$$

To find the loss in branch $2 \Delta s_{2 \mathrm{i} 4 \mathrm{t} 1}$ caused by RC $I_{4 \mathrm{t} 1}$, current $I_{2 J 4 \mathrm{o}}$ in expression (9) must be substituted by $I_{2 \mathrm{i} 4 \mathrm{t} 1}$ defined by (10), which flows in the branch.

The voltage drop across some branch is a linear function of current and can be easily found if the current is known. E.g. voltage drop $\Delta u_{2 \mathrm{i} 4 \mathrm{t} 1}$ across branch 2 from RC $I_{4 \mathrm{t} 1}$ is as follows:

$$
\Delta u_{2 \mathrm{i} 4 \mathrm{t} 1}=Z_{2} I_{2 \mathrm{i} 4 \mathrm{i} 1}
$$

and equals $-133-791 i$; this current is negative because in Fig. 2 current $I_{2 \mathrm{i} 4 \mathrm{t} 1}$ flows in a direction opposite to the one in impedance $Z_{2}$. The voltage drop of the total current in branch 2 is $1988.4+2801.3 i$.

These secondary quantities show the influence of the supplier or consumer on the mode of the grid and indicate severe overloads of grid elements and their origin.

\section{OPTIMUM USE OF POWER SUPPLIERS}

Among other factors, losses depend on the distribution of power required by the consumers between the suppliers [14]. It can be said that if the price is equal for all the suppliers, then, the farther the supplier is located, the less electricity has to be bought to reduce the losses in the grid. It was found that an optimal load distribution occurs when the active components $U_{\mathrm{d}}$ of the distinctive voltages mentioned here on the way from the supplier to the generalized load (see Fig. 4) are equal for all the suppliers.

The distinctive voltages of suppliers $4 ; 5 ; 7$ are defined as follows:

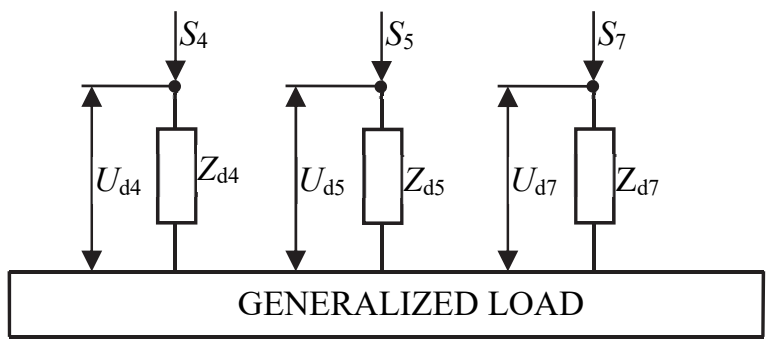

Fig. 4. Representation of the grid with the view of its optimization.

$$
U_{\mathrm{d} 4}=\Delta s_{4} /\left|J_{4}\right| ; \quad U_{\mathrm{d} 5}=\Delta s_{5} /\left|J_{5}\right| ; \quad U_{\mathrm{d} 7}=\Delta s_{7} /\left|J_{7}\right|,
$$

where $\Delta s_{4} ; \Delta s_{5} ; \ldots ; \Delta s_{7}$ are the power losses due to the respective suppliers:

$$
\begin{gathered}
\Delta s_{4}=\Delta s_{4 \mathrm{t} 1}+\Delta s_{4 \mathrm{t} 2}+\Delta s_{4 \mathrm{t} 3}+\Delta s_{4 \mathrm{t} 6} ; \quad \Delta s_{5}=\Delta s_{5 \mathrm{t} 1} \ldots \\
\Delta s_{7}=\Delta s_{7 \mathrm{t} 1} \ldots
\end{gathered}
$$

Summary loss $\Delta s$ is shown in Table VI with appropriate (different) power distributions (see Table I) between the suppliers, which shows that power losses are minimal when the active components of distinctive voltages are equal or close to each other.

It can be seen that the closer the active components of the distinctive voltages each other, the less the active losses in the grid.

TABLE VI

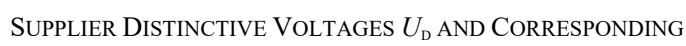
ACTIVE LOSS $\Delta P$

\begin{tabular}{|c|c|c|c|c|}
\hline Case & $U_{\mathrm{d} 4}$ & $U_{\mathrm{d} 5}$ & $U_{\mathrm{d} 7}$ & $\Delta p$ \\
\hline $\mathrm{Qa}$ & $1163+3583 i$ & $237+594 i$ & $600+3120 i$ & 337130 \\
\hline $\mathrm{Qc}$ & $769+2313 i$ & $550+1605 i$ & $614+3172 i$ & 288265 \\
\hline $\mathrm{Qc} 1$ & $531+1545 i$ & $736+2207 i$ & $618+3187 i$ & 287684 \\
\hline $\mathrm{Qc} 2$ & $650+1929 i$ & $643+1906 i$ & $616+3179 i$ & 285161 \\
\hline $\mathrm{Fa}$ & $2562+1704$ & $894+5696 i$ & $248+1233 i$ & 552510 \\
& $8 i$ & & & \\
\hline $\mathrm{Fc}$ & $1500+1003$ & $1717+10799 i$ & $251+1243 i$ & 443389 \\
\hline $\mathrm{Fc} 1$ & $1140+7636 i$ & $1217+7658 i$ & $317+1526 i$ & 286719 \\
\hline $\mathrm{Fc} 2$ & $724+4868 i$ & $755+4751 i$ & $384+1812 i$ & 201245 \\
\hline Fc3 & $536+3611 i$ & $546+3438 i$ & $415+1942 i$ & 185956 \\
\hline Fc4 & $380+2573 i$ & $390+2457 i$ & $439+2044 i$ & 184200 \\
\hline & & & & \\
\hline Fc5 & $463+3128 i$ & $464+2921 i$ & $427+1993 i$ & 183942 \\
\hline
\end{tabular}

Negative RLs quickly disappear when the distinctive voltages approach is used. In case Qa, we have three negative 
RL values (Table III) while in case Qc, there are none of them. In case $\mathrm{Fa}$, there is only one negative RL value (Table $\mathrm{V}$ ) but it persists in case Fc and Fc1 and disappears only in Fc2. So, the total loss $\Delta p$ is greater when there are negative RLs.

Distinctive impedances

$$
Z_{\mathrm{d} 4}=\Delta s_{4} /\left|J_{4}\right|^{2} ; Z_{\mathrm{d} 5}=\Delta s_{5} /\left|J_{5}\right|^{2} ; Z_{\mathrm{d} 7}=\Delta s_{7} /\left|J_{7}\right|^{2}
$$

vary relatively little when loads change:

Qa: $Z_{\mathrm{d} 4}=6.56+20.21 i ; Z_{\mathrm{d} 5}=2.68+6.69 ; Z_{\mathrm{d} 7}=3.37+17.02 i$; Qc2: $Z_{\mathrm{d} 4}=5.43+16.1 i ; Z_{\mathrm{d} 5}=4.4+13.04 i ; Z_{\mathrm{d} 7}=3.35+17.3 i$.

For a closed grid:

Fa: $Z_{\mathrm{d} 4}=14.78+98.37 i ; Z_{\mathrm{d} 5}=12.8+81.6 i ; Z_{\mathrm{d} 7}=1.34+6.64 i ;$

Fc5: $\quad Z_{\mathrm{d} 4}=16.81+113.51 i ; \quad Z_{\mathrm{d} 5}=13.66+86.03 i ;$ $Z_{\mathrm{d} 7}=1.17+5.47 i$.

On the basis of this feature, it can be said that the power loss of a supplier is approximately proportional to the square of the supplier's power.

\section{QUick Determination OF Node Voltages}

When the current of a supplier node changes, the voltage in other nodes changes in the same direction; when change takes place at a consumer node, the change takes place in the opposite direction. The voltage change takes place almost linearly to power change. The sensitivity of any node voltage to power of any other node depends on how far apart electrically they are located.

At first, the most common mode of a power system (the basic mode) should be chosen and the node voltages $U_{v}$ should be calculated by DA. The second mode (auxiliary) should be calculated by DA as well. Using these two modes, the sensitivity factors should be obtained, which are used for quick calculation of node voltages of other (eventual) mode of a grid. The eventual mode is the mode when the current of some node differs from that of the basic mode.

In Fig. 5 the circuit diagram is shown, explaining the idea.
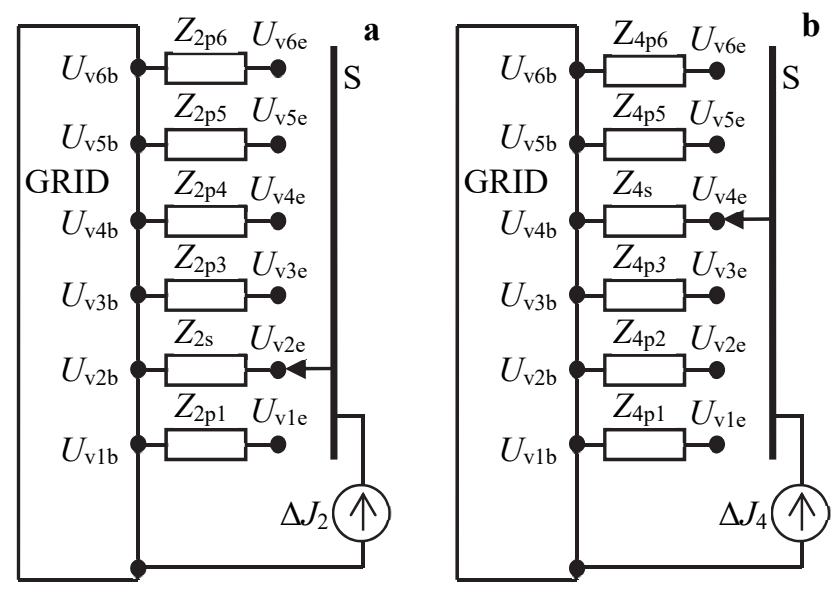

Fig. 5. Updating the voltages of a grid (a) as a result of change of consumer current $J_{2}$; (b) change of supplier current $J_{4}$.

The power system for this purpose can be considered as a collection of voltage sources [15]. It can be represented by its no-load voltage $U_{\text {vib }}(i$ stands for $1 \ldots 6, b$ - for 'basic') and by the appropriate impedance. $\mathrm{U}_{\mathrm{v} 7}$, which is shown in Table II, is not needed since it is slack bus voltage. Impedance $Z$ (putative impedance) for nodes 4 and 2, along with other data, is given in Tables VII and VIII.

\section{TABLE VII}

DATA of AUXILIARY CASE Q4I3 FOR QUiCK UPDATE (IMPACT OF NODE 4)

\begin{tabular}{|c|c|c|c|}
\hline No & $J_{1 \ldots 6}$ & $U_{\mathrm{v} 1 \ldots 6}$ & $Z_{i p k}$ \\
\hline 1 & $\begin{array}{c}69.99- \\
0.086 i\end{array}$ & $190500.4-244.3 i$ & $Z_{4 \mathrm{p} 1}=0.939+3.255 i$ \\
& & \\
\hline 2 & 279.45 & $190774.8-397.8 i$ & $Z_{4 \mathrm{p} 2}=7.747+33.195 i$ \\
& $-530.0 i$ & & \\
\hline 3 & 34.78 & $194009.8+4947.2 i$ & $Z_{4 \mathrm{p} 3}=15.439+57.338 i$ \\
& $-16.29 i$ & & \\
\hline 4 & 276.09 & $194942.1+6401.3 i$ & $Z_{4 s}=17.221+63.098 i$ \\
& $-76.43 i$ & & \\
\hline 5 & $87.15-$ & $191319.7+917.8 i$ & $Z_{4 \mathrm{p} 5}=7.566+33.014 i$ \\
& $8.29 i$ & & \\
\hline 6 & $52.38-$ & $190924.9-29.6 i$ & $Z_{4 \mathrm{p} 6}=4.467+31.727 i$ \\
& $5.25 i$ & & \\
\hline
\end{tabular}

In Table VII, putative impedances are given for the supplier node and are positive.

\section{TABLE VIII}

DATA OF AUXILIARY CASE Q2I FOR QUICK UPDATE (IMPACT OF NODE 2)

\begin{tabular}{|c|c|c|c|}
\hline No & $J_{1 \ldots 6}$ & $U_{v 1 \ldots 6}$ & $Z_{\text {ipk }}$ \\
\hline 1 & $70.14-0.29 i$ & $190080-780 i$ & $Z_{2 \mathrm{pl}}=-0.924-3.257 i$ \\
\hline 2 & $368.15-81.14 i$ & $187030-5910 i$ & $Z_{2 \mathrm{~s}}=-5.667-31.652 i$ \\
\hline 3 & $35.16-18.19 i$ & $188270-2590 i$ & $\mathrm{Z}_{2 \mathrm{p} 3}=-5.096-31.569 i$ \\
\hline 4 & $176.39-26.24 i$ & $188740-1620 i$ & $\mathrm{Z}_{2 \mathrm{p} 4}=-4.875-31.505 i$ \\
\hline 5 & $88.56-11.05 i$ & $187620-4590 i$ & $\mathrm{Z}_{2 \mathrm{p} 5}=-5.466-31.648 i$ \\
\hline 6 & $53.22-6.92 i$ & $187190-5540 i$ & $\mathrm{Z}_{2 \mathrm{p} 6}=-5.641-31.653 i$ \\
\hline
\end{tabular}

In Table VIII, they are given for the consumer node and are negative. When the grid mode is basic, the current change $\Delta J=0$ and the eventual voltage on the outlet $U_{\mathrm{ve}}=U_{\mathrm{vb}}$; when the current of some node changes (eventual mode), the current change $\Delta J \neq 0$, this current is fed to the outlet, a voltage drop across the putative impedance appears and the eventual voltage $U_{\mathrm{ve}}$ is not equal to the basic one. Putative impedance is designated $Z_{i \text { s }}$ when voltage is considered on that very node $i$ where current $\Delta J_{\mathrm{i}}$ appears; it is designated $Z_{i \mathrm{p} k}$ when the voltage $U_{v k}$ on some (other than $i$ ) node $k$ of this grid is considered.

Eventual voltage $U_{\mathrm{v} i}$ or $U_{\mathrm{v} k}$ of node $i$ or $k$ is calculated, imagining that current change $\Delta J_{i}$ in node $i$ is fed to this very node $i$ through switch $\mathrm{S}$.

To find putative impedances, node voltages $U_{\mathrm{v} 1 \mathrm{~b}}, \ldots, U_{\mathrm{v} 6 \mathrm{~b}}$, $U_{\mathrm{vla}}, \ldots, U_{\mathrm{v} 6 \mathrm{a}}$ and node currents $J_{1 \mathrm{~b}}, \ldots, J_{6 \mathrm{~b}} ; J_{1 \mathrm{a}}, \ldots, J_{6 \mathrm{a}}$ in the basic and auxiliary mode of the grid should be obtained by DA.

Then impedances $Z_{i \mathrm{~s}}$ and $Z_{i \mathrm{p} k}$ are defined as follows:

$$
Z_{i \mathrm{~s}}=\frac{U_{\mathrm{via}}-U_{\mathrm{vib}}}{J_{\mathrm{via}}-J_{\mathrm{vib}}} ; Z_{i \mathrm{p} k}=\frac{U_{\mathrm{vka}}-U_{\mathrm{vkb}}}{J_{\mathrm{via}}-J_{\mathrm{vib}}} .
$$

Naturally, the value of $Z_{i \mathrm{~s}}$ is greater than the value of any $Z_{i \mathrm{p} k}$ since the voltage at this node $i$ is the most sensitive to its current. These impedances show which nodes are more prone to voltage variations. 
Eventual $U_{\mathrm{v} i} ; U_{\mathrm{v} k}$ voltages are found by the following expressions:

$$
U_{\mathrm{vie}}=U_{\mathrm{vib}}+Z_{i \mathrm{~s}}\left(J_{i \mathrm{e}}-J_{i \mathrm{~b}}\right) ; U_{\mathrm{vke}}=U_{\mathrm{vkb}}+Z_{i \mathrm{pk}}\left(J_{i \mathrm{e}}-J_{i \mathrm{~b}}\right) .
$$

This time, $J_{i e}$ is not obtained by DA because we do not use DA or any other program (as it is inconvenient at the moment) but use the quick method, using the known basic voltage $U_{\mathrm{vb}}$ and the changed power $S_{i \mathrm{e}}$ :

$$
J_{i \mathrm{e}}=\frac{\hat{S}_{i \mathrm{e}}}{3 \hat{U}_{\mathrm{vib}}} .
$$

If there is doubt about the accuracy, the node current $J_{i \mathrm{e}}$ can be recalculated by (17) using the voltage $U_{\text {vie }}$ obtained by (16):

TABLE IX

THE ACCURACY OF QUick CALCUlation of Node Voltage CHANGES

\begin{tabular}{|c|c|c|c|c|c|c|}
\hline \multirow{2}{*}{$\begin{array}{c}\text { Case } \\
\text { base/auxil./event. }\end{array}$} & $d_{1 \mathrm{e}}$ & $d_{2 \mathrm{e}}$ & $d_{3 \mathrm{e}}$ & $d_{4 \mathrm{e}}$ & $d_{5 \mathrm{e}}$ & $d_{6 \mathrm{e}}$ \\
\cline { 2 - 7 } & $d_{1 \mathrm{e}}{ }^{(1)}$ & $d_{2 \mathrm{e}}{ }^{(1)}$ & $d_{3 \mathrm{e}}{ }^{(1)}$ & $d_{4 \mathrm{e}}{ }^{(1)}$ & $\mathrm{d}_{5 \mathrm{e}}{ }^{(1)}$ & $d_{6 \mathrm{e}}{ }^{(1)}$ \\
\hline & 0.0460 & 0.0771 & 0.0765 & 0.0780 & 0.0756 & 0.0757 \\
\cline { 2 - 7 } & 0.0577 & 0.0053 & 0.0018 & 0.0021 & 0.0040 & 0.0052 \\
\hline & 0.0981 & 0.0188 & 0.0375 & 0.0472 & 0.0226 & 0.0197 \\
\cline { 2 - 7 } & 0.0576 & 0.0472 & 0.0759 & 0.0864 & 0.0578 & 0.0496 \\
\hline & 0.0337 & 0.0602 & 0.0261 & 0.0215 & 0.0488 & 0.0744 \\
\cline { 2 - 7 } & 0.0856 & 0.0112 & 0.0732 & 0.0661 & 0.0960 & 0.0439 \\
\hline & 0.0332 & 0.0102 & 0.0193 & 0.0969 & 0.0331 & 0.0115 \\
\cline { 2 - 7 } & 0.0299 & 0.0098 & 0.0214 & 0.0995 & 0.0357 & 0.0120 \\
\hline & 0.0459 & 0.0461 & 0.0454 & 0.0453 & 0.0467 & 0.0460 \\
\cline { 2 - 7 } & 0.0009 & 0.0031 & 0.0021 & 0.0021 & 0.0047 & 0.0071 \\
\hline
\end{tabular}

$d_{n \mathrm{e}}$ is the value computed by $(17) ; d_{n \mathrm{e}}{ }^{(1)}-$ the value computed by (18).

$$
J_{i \mathrm{e}}{ }^{(1)}=\frac{\hat{S}_{\mathrm{ie}}}{3 \hat{U}_{\mathrm{vie}}} .
$$

and the node voltages are calculated again by (16).

To check the accuracy of this method, in this paper eventual modes were calculated also by DA and node voltages $U_{\text {vieDA, }}$, $U_{\text {vkeDA }}$ were obtained. For any node $n$, accuracy is defined as the difference between voltage $U_{\mathrm{v} n}$ obtained by the quick method and voltage $U_{\mathrm{v} n \mathrm{eDA}}$ obtained by DA; this difference is related to the difference between voltage $U_{\mathrm{v} n \mathrm{EA}}$ and basic mode voltage $U_{\mathrm{v} n \mathrm{~b}}$ :

$$
d_{n}=\frac{\left|U_{\mathrm{v} n \mathrm{e}}-U_{\mathrm{v} n e \mathrm{DA}}\right|}{\left|U_{\mathrm{v} n e \mathrm{DA}}-U_{\mathrm{v} n \mathrm{~b}}\right|} .
$$

The accuracy results based on (17) and (18) are shown in Table IX. In some cases, the recalculated values are more accurate.

\section{CONCLUSION}

1. Negative reciprocal losses indicate increased loss in the grid; the more often they appear, the greater is the total power loss in a grid. Negative losses can be removed and grid losses reduced by a different distribution of power between suppliers.

2. Using the DA algorithm, the power loss and the voltage drop can be determined for any branch of the grid and for any current, be this current caused by any node current or by some reciprocal current.

3. In a grid with minimal losses, the active components of distinctive voltages are equal and the distribution of the supplier power is optimal.

4. For any supplier, distinctive impedance can be obtained, which can be used for quick estimation of the power losses caused by the suppliers.

5. Using putative impedances, the grid node voltages can be updated quickly.

\section{REFERENCES}

[1] Electricity trading on the open market. [Online]. Available: www.kp.gov.lv

[2] J. Gerhards, A. Mahnitko, and B. Papkovs, Control, Optimization of Power Grids and the Risks. Riga: RTU, 2011, pp. 150-154.

[3] Simulator Overview "Power World". [Online]. Available: www.powerwold.com/produkts/simulator

[4] V. Kazancev, Loss of Electricity in the Networks of Electrical Grids, Moscow: Energopublication, 1983.

[5] A. Vanags, Electrical Networks and Grids, Riga: RTU, 2007, pp. 156174.

[6] O. Ozdemir, F. D. Munoz, J. L. Ho, and B. F. Hobbs, "Economic Analysis of Transmission Expansion Planning With Price-Responsive Demand and Quadratic Losses by Successive LP," IEEE Transactions on Power Systems, vol. 31, no. 2, pp. 1096-1107, Mar. 2016. https://doi.org/10.1109/tpwrs.2015.2427799

[7] J.-H. Teng, "Power flow and loss allocation for deregulated transmission systems," International Journal of Electrical Power \& Energy Systems, vol. 27, no. 4, pp. 327-333, May 2005. https://doi.org/10.1016/j.ijepes.2004.12.005

[8] M. Atanasovski and R. Taleski, "Power Summation Method for Loss Allocation in Radial Distribution Networks With DG," IEEE Transactions on Power Systems, vol. 26, no. 4, pp. 2491-2499, Nov. 2011. https://doi.org/10.1109/tpwrs.2011.2153216

[9] A. J. Conejo, J. M. Arroyo, N. Alguacil, and A. L. Guijarro, "Transmission loss allocation: a comparison of different practical algorithms," IEEE Transactions on Power Systems, vol. 17, no. 3, pp. 571-576, Aug. 2002. https://doi.org/10.1109/tpwrs.2002.800894

[10] J. Survilo, "Reciprocal Load-Generator Power Losses in a Grid," Latvian Journal of Physics and Technical Sciences, 2013, No. 6, vol. 50, pp. 4863.

[11] J. Survilo, "Account of Losses in Electricity Sales," in Riga Technical University 54th International Scientific Conference: Digest Book and Electronic Proceedings, 2013, pp. 77-79.

[12] J. Survilo, "Determination of reciprocal current by electricity delivery to more than one consumer," in 2014 55th International Scientific Conference on Power and Electrical Engineering of Riga Technical University (RTUCON), Oct. 2014. https://doi.org/10.1109/rtucon.2014.6998183

[13] J. Survilo, "A method for node prices formation," in 2015 56th International Scientific Conference on Power and Electrical Engineering of Riga Technical University (RTUCON), Oct. 2015. pp. 283-288. https://doi.org/10.1109/rtucon.2015.7343121

[14] K. Mahmoud, N. Yorino, and A. Ahmed, "Optimal Distributed Generation Allocation in Distribution Systems for Loss Minimization," IEEE Transactions on Power Systems, vol. 31, no. 2, pp. 960-969, Mar. 2016. https://doi.org/10.1109/tpwrs.2015.2418333

[15] L. Neiman and K. Demirchian, Theoretical Foundations of Electrical Engineering. Leningrad: Energo Publishing House, 1981, p. 128. 